\title{
Tomographic retrieval of cloud liquid water fields from a single scanning microwave radiometer aboard a moving platform - Part 1: Field trial results from the Wakasa Bay experiment
}

\author{
D. Huang ${ }^{1}$, A. J. Gasiewski ${ }^{2}$, and W. Wiscombe ${ }^{1,3}$ \\ ${ }^{1}$ Brookhaven National Laboratory, Upton, NY 11973, USA \\ ${ }^{2}$ University of Colorado, Boulder, CO 80309, USA \\ ${ }^{3}$ NASA Goddard Space Flight Center (code 913), Greenbelt, MD 20771, USA
}

Received: 14 April 2009 - Published in Atmos. Chem. Phys. Discuss.: 15 May 2009

Revised: 1 July 2010 - Accepted: 10 July 2010 - Published: 22 July 2010

\begin{abstract}
Tomographic methods offer great potential for retrieving three-dimensional spatial distributions of cloud liquid water from radiometric observations by passive microwave sensors. Fixed tomographic systems require multiple radiometers, while mobile systems can use just a single radiometer. Part 1 (this paper) examines the results from a limited cloud tomography trial with a single-radiometer airborne system carried out as part of the 2003 AMSR-E validation campaign over Wakasa Bay of the Sea of Japan. During this trial, the Polarimetric Scanning Radiometer (PSR) and Microwave Imaging Radiometer (MIR) aboard the NASA P3 research aircraft provided a useful dataset for testing the cloud tomography method over a system of low-level clouds. We do tomographic retrievals with a constrained inversion algorithm using three configurations: PSR, MIR, and combined PSR and MIR data. The liquid water paths from the PSR retrieval are consistent with those from the MIR retrieval. The retrieved cloud field based on the combined data appears to be physically plausible and consistent with the cloud image obtained by a cloud radar. We find that some vertically-uniform clouds appear at high altitudes in the retrieved field where the radar shows clear sky. This is likely due to the sub-optimal data collection strategy. This sets the stage for Part 2 of this study that aims to define optimal data collection strategies using observation system simulation experiments.
\end{abstract}

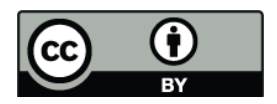

Correspondence to: D. Huang (dhuang@bnl.gov)

\section{Introduction}

The study of climate and climate change has long been hindered by a poor understanding of the effect of clouds (Ramanathan et al., 1989). The most important characteristic of clouds after their simple presence or absence is their liquid water content (LWC). Cloud microwave tomography, first proposed by Warner et al. (1985), permits the retrieval of LWC spatial distributions from microwave radiometric observations. This method retrieves the spatial structure of LWC by observing the cloud's thermal emission from multiple directions at distinct locations. A certain degree of intersection between microwave beams is necessary for the cloud tomography method to be successful.

One configuration proposed by Warner et al. (1985) is shown schematically in Fig. 1. An aircraft carries a single upward-looking radiometer, which switches automatically between two fixed-direction antennas, and flies along a horizontal line just under a cloud. A theoretical study of this configuration (Drake and Warner, 1988) showed that it performs similarly to a ground-based configuration that uses two distinctly-located scanning microwave radiometers. A field test was carried out in Louisiana and the retrieved LWC agreed statistically with that measured in situ (Warner and Drake, 1988).

There were many limitations that inhibited the practical use of cloud tomography in the 1980s. Chief among them were the high cost and huge size of radiometers, and their slow scanning rate. Microwave technologies have advanced considerably and microwave radiometers have become more portable and reliable while costs have fallen, owing to the

Published by Copernicus Publications on behalf of the European Geosciences Union. 


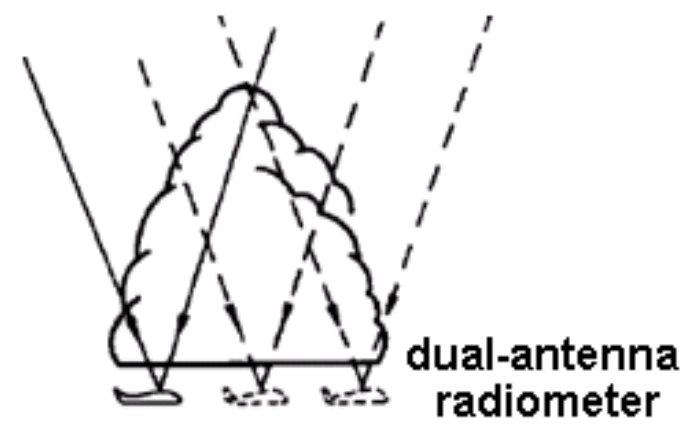

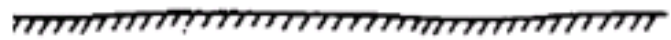

Fig. 1. An airborne cloud tomography configuration proposed by Warner et al. (1985). The aircraft carries a single upwardlooking radiometer, which switches automatically between two fixed-direction antennas, and flies along a horizontal line just under a cloud.

commercialization of microwave remote sensing. For example, Padmanabhan et al. (2009) showed that monolithic microwave integrated circuit technology and unique packaging yields a compact, inexpensive and low-power instrument that can be deployed as a network to tomographically retrieve three-dimensional fields of water vapor. The reduction in radiometer size with increasing reliability finally makes airborne scanning microwave radiometers reachable for the community. At the same time, we can bring many more computational resources to bear upon the mathematical problem of cloud tomography than in Warner's day, e.g., faster computers, better cloud models (used in data assimilation mode), and more advanced mathematical tools. Now is the time to take advantage of the hardware and computational advances to turn cloud tomography into a ubiquitous tool for cloud physics.

Huang et al. (2008a, b) started the theoretical reexamination of the cloud tomography method after its twodecade dormancy. The first paper rigorously examined the underlying mathematical problem using observation system simulation experiments based on large eddy simulation. It was revealed that, like other limited angle tomography problems, the mathematical problem of cloud tomography with limited number of ground radiometers (2-4) is highly illposed. Its solution is non-unique and very sensitive to observational noise and numerical errors. The second paper then focused on the development of tomographic retrieval algorithms that make use of various types of constraints based on a priori knowledge to improve the retrieval. It was demonstrated that appropriate constraints can improve the accuracy of the tomographic retrieval by one order of magnitude.

The ground-based cloud tomography configuration has the advantage of collecting data without human intervention and thus is suitable for long-term cloud observation. The airborne configuration is more flexible, allowing cloud-chasing, but has poor temporal coverage and is usually costly to deploy. However, the airborne configuration with dual antennas (Fig. 1) also has limitations, e.g., very limited observing angles and technical difficulties in building such dualantenna radiometers. The new generation of scanning radiometers developed by the NOAA-CU Center for Environmental Technology (CET) at University of Colorado at Boulder, called Polarimetric Scanning Radiometer (PSR), provides a good opportunity to re-evaluate the capability of the airborne cloud tomography method with state-of-the-art microwave techniques. The PSR was deployed on the NASA P-3 research aircraft along with several other microwave remote sensing instruments during the 2003 Advanced Microwave Scanning Radiometer for EOS (AMSR-E) validation campaign over Wakasa Bay of the Sea of Japan.

In Part 1 (this paper) we examine the results from the limited cloud tomography trial during the 2003 AMSR-E campaign in which the PSR scanned through a system of lowlevel cloud layers. In Part 2, we then conduct observation system simulation experiments about the mobile cloud tomography system and present several recommendations on how to improve the mobile system.

The paper is organized as follows. Section 2 provides a detailed description of the data collected during the Wakasa Bay experiment. Section 3 sets up the theoretical basis for the tomographic inversion problem. Section 4 elaborates the retrieval procedures. Section 5 presents the retrieval results and also focuses on validation of the tomographic retrievals. Section 6 summarizes the findings of this paper.

\section{Data}

The data used in this study were collected during the 2003 AMSR-E campaign at Wakasa Bay from the instrumented NASA P-3 research aircraft. The instruments included the PSR scanning microwave radiometer with multiple measurement frequencies from 10.7 to $89 \mathrm{GHz}$, a high frequency Microwave Imaging Radiometer (MIR) covering the spectral range from 89 to $340 \mathrm{GHz}$, and an Airborne Cloud Radar (ACR) operating at $95 \mathrm{GHz}$ (see detailed specifications in Table 1).

\subsection{Description of the Wakasa Bay field campaign}

The 2003 Wakasa Bay field campaign was a cooperative effort between the Japan Aerospace Exploration Agency (JAXA), and NASA AMSR-E teams focusing on the physical validation of shallow snowfall and rainfall retrievals from the AMSR and AMSR-E (Lobl et al., 2007). Wakasa Bay, on the eastern end of the Sea of Japan, has fairly predictable cold air outbreaks during winter in which cold air from the Eurasian continent blows over the relatively warm Sea of Japan. These storms typically produce very shallow rainfall layers near the 
Table 1. Instrument specifications and operation modes during the Wakasa Bay cloud tomography experiment.

\begin{tabular}{|c|c|c|c|}
\hline Instrument & Frequency & Beamwidth & Scan mode \\
\hline PSR & $\begin{array}{l}10.7,18.7,21.5,37 \text {, and } \\
89 \mathrm{GHz}, 9.6-11.5 \mu\end{array}$ & $2.3^{\circ}$ at $37 \mathrm{GHz}$ & $\begin{array}{l}\text { Along-track } \\
\text { scan }\end{array}$ \\
\hline MIR & $\begin{array}{l}89,150,183 \pm 1,3,7 \\
220, \text { and } 340 \mathrm{GHz}\end{array}$ & $3.5^{\circ}$ at $89 \mathrm{GHz}$ & $\begin{array}{l}\text { Cross-track } \\
\text { scan }\end{array}$ \\
\hline ACR & $95 \mathrm{GHz}$ & $0.56^{\circ}$ & Nadir staring \\
\hline
\end{tabular}

surface, where warm boundary layer air mixes with the cold air aloft.

The validation campaign was intended to improve understanding of winter precipitation over mid-latitude oceans. During the three-week experiment from 14 January to 3 February 2003, the team collected a wealth of now publicly available data of low- and mid-altitude winter clouds and precipitation. The data relevant to this study are ground and airborne radar reflectivities, microwave brightness temperatures, and dropsonde data. To test the validity of the airborne cloud tomography method, we selected a 176-km long flight line (Fig. 2) obtained on January 28, 2003 starting from $\left(37.525^{\circ} \mathrm{N}, 133.522^{\circ} \mathrm{E}\right)$ to $\left(38.8^{\circ} \mathrm{N}, 134.792^{\circ} \mathrm{E}\right)$, within which the PSR had appropriate observational geometry for the purpose of cloud tomography retrieval (see more details in Sect. 2.2).

\subsection{PSR data}

The PSR developed by CET is a versatile microwave radiometer for imaging the polarimetric microwave emission of the Earth's oceans, land, ice, clouds, and precipitation. The PSR provides vertical and horizontal polarization observations from C-band to $\mathrm{W}$-band frequencies (Piepmeier and Gasiewski, 1996). The basic concept of the PSR is a set of polarimetric radiometers housed within a gimbal-mounted scanhead drum. The scanhead drum is rotatable by the gimbal positioner so that the radiometers can view any angle within $\pm 70^{\circ}$ elevation off nadir at any azimuthal angle (a total of $1.32 \mathrm{sr}$ solid angle), as well as external hot and ambient calibration targets. The PSR configuration was operated in various scanning modes including conical, cross-track, along-track, fixed-angle stare, and spotlight modes during the Wakasa Bay field campaign. Figure 3 shows the swaths of three successive PSR along-track scans; each covers an isosceles-trapezoid-shaped region whose base angle is $20^{\circ}$ and the distance between two successive scan cycles is approximately $5.9 \mathrm{~km}$. Note that the swath of each scan cycle is shown in different color. The lower parts of two successive scans have significant overlap with each other, while the upper parts have almost no overlap. Furthermore, each scan cycle has a little overlap with its second successive scan and no overlap with its third successive scan (even at low alti- tudes). As it will be shown later in this paper, the poor overlap between the scan cycles substantially limits the capability of the mobile cloud tomography technique to retrieve smallscale cloud structure.

The brightness temperature data are obtained using a data processing and calibration algorithm developed by CET. The first step is decoding the navigation information such as geographic coordinates, altitude, yaw, roll, and pitch of the platform from the recorded navigation data. Then the radiometric data are partitioned into individual "maneuvers" based on the navigation information - a maneuver is a segment of flight during which the aircraft keeps the same altitude and orientation and the radiometer remains in the same scanning mode. The calibration algorithm described in Corbella et al. (2002) is used to calculate the radiometer offset and gain factors. The calibration method consists of periodically switching each of the receivers to view its corresponding noise diode and infrequently viewing two external reference targets that have up to a $100-{ }^{\circ} \mathrm{C}$ temperature difference. The gain and offset at closely spaced time intervals are computed using the noise diodes, and by referring the diodes' noise temperatures to precise measurements of the external targets.

The original PSR data were recorded with 7.5-millisecond integration time, resulting in about 8000 scan angles per oneminute scan. The 7.5-millisecond integration time corresponds to a $0.03^{\circ}$ angular increment between two successive beams. Given the $2.3^{\circ}$ antenna beam width, the actual beam volumes of any two successive beams are $99 \%$ overlapped and thus the redundancy in the original data is very high. We thus average the original data to 150-millisecond resolution not only to reduce the total volume of the data but also to average down the random integration noise in the data.

Horizontally-polarized microwave emission from the sea surface has a maximum in the zenith direction and decreases with incidence angle (bell-shaped), while verticallypolarized emission increases with incidence angle (bowlshaped). On the other hand, microwave emission from cloud liquid water shows no or very weak polarization signal. Thus, averaging the measured brightness temperatures over the two polarizations will suppress the range of angular variation due to the background sea surface emission (thus reducing the uncertainty associated with the sea surface emission model) and will enhance the signal-to-noise ratio of the tomographic data. Figure 4a shows the brightness temperatures at $37 \mathrm{GHz}$ averaged over the two polarizations as a function of distance (or, equivalently, UTC time). The brightness temperatures range from $160 \mathrm{~K}$ to $220 \mathrm{~K}$. Figure $4 \mathrm{~b}$ shows that the corresponding view angles range from $70^{\circ}\left(70^{\circ}\right.$ from nadir in the forward direction) to $-70^{\circ}\left(70^{\circ}\right.$ from nadir in the backward direction). Periodic observation gaps are noticeable in the data. These gaps correspond to the portion of the PSR scan cycles in which no atmosphere observations were made when the scanhead was viewing or preparing to view the external blackbody calibration targets. 


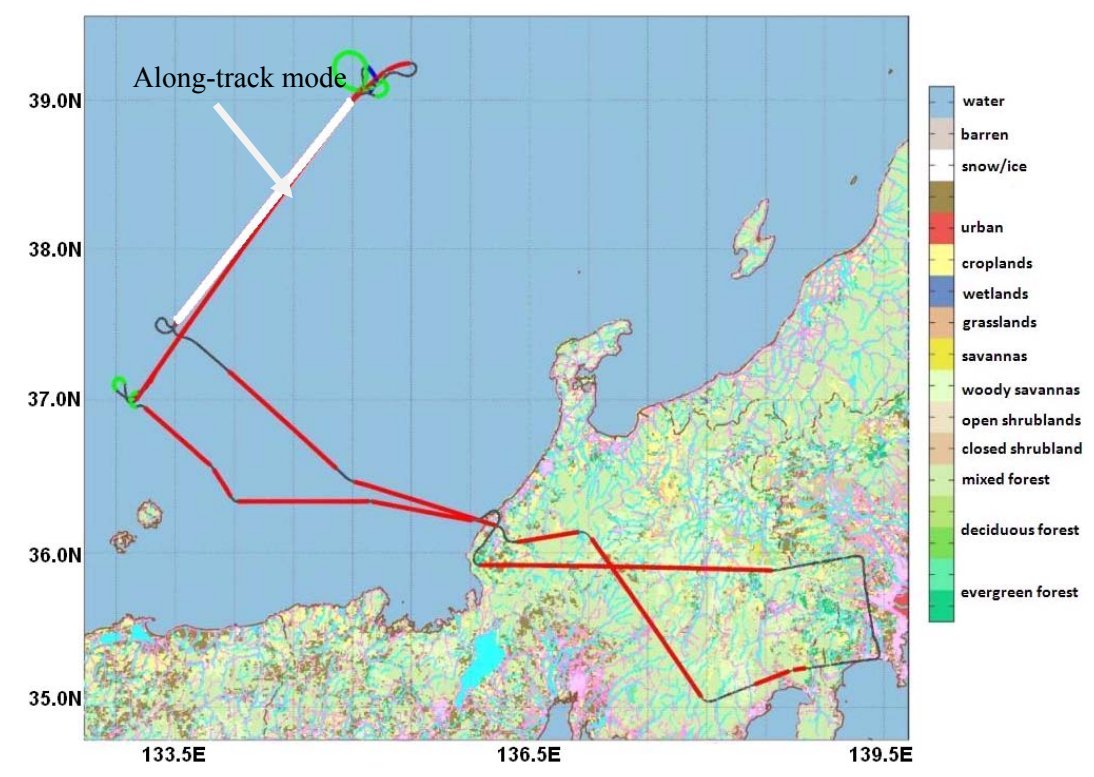

Fig. 2. Flight tracks of the NASA P-3 aircraft on 28 January 2003 over the Sea of Japan. White - PSR operated on the along-track mode (the only mode suitable for cloud tomography retrieval); Red - the aircraft flew in a straight and level line; Green - the aircraft flew in a constant angle turns.

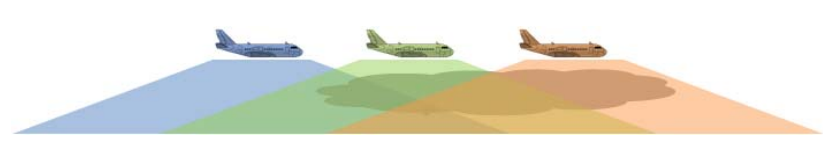

Fig. 3. Schematic of the geometry of the PSR along-track scans. The cartoon shows three successive along-track scan cycles, each shown in a different color. Each PSR swath spans $\pm 70^{\circ}$ off the nadir. The aircraft translates approximately $5.9 \mathrm{~km}$ during a full PSR scan cycle. It can be seen from the cartoon that a scan cycle moderately overlaps with its successive scan, but hardly overlaps with its second successive scan.

\subsection{MIR data}

The MIR is a nine-channel airborne imaging radiometer designed to fly aboard high altitude aircraft for atmospheric research (Racette et al., 1996). Three dual pass band channels are centered on the strongly opaque $183 \mathrm{GHz}$ water absorption line and a fourth channel is located at $150 \mathrm{GHz}$. These channels have varying degrees of opacity from which the water vapor profile can be inferred. There are two additional channels located at $89 \mathrm{GHz}$ and $220 \mathrm{GHz}$. The radiometer has a $3.5^{\circ}$ beamwidth for most of its channels. During the Wakasa Bay field campaign, the imager was programmed to operate in the cross-track scan mode with a $106^{\circ}$ swath. In each three-second scan cycles, it views two external calibration targets in addition to the $106^{\circ}$ scene scan; one of these targets is heated to a temperature of $330 \mathrm{~K}$ and the other remains at the ambient temperature of the aircraft cruising altitude.
The MIR data from the Wakasa Bay field campaign were calibrated by the Microwave Sensors Branch of NASA/Goddard Space Flight Center and were made available at National Snow and Ice Data Center (NSIDC) (Wang et al., 2004). We extract the cross-track nadir-looking beams from the MIR data archive. The reason for choosing only the nadir beams is that these beams are located in the vertical plane of the PSR along-track scans and thus can serve as a useful supplement to the along-track PSR data that contain many observation gaps (each $1-3 \mathrm{~km}$ long, as shown in Fig. 4a). To minimize the complexity caused by scattering of ice particles, we use the lowest frequency of MIR, i.e., $89 \mathrm{GHz}$.

The minimum brightness temperature observed by MIR at $89 \mathrm{GHz}$ (Fig. 4c) is $195 \mathrm{~K}$, possibly corresponding to a clear sky condition; the maximum value observed is $250 \mathrm{~K}$, indicating the presence of a moderately thick cloud. The MIR brightness temperatures at $89 \mathrm{GHz}$, on average, are about $30 \mathrm{~K}$ higher than those of the PSR at $37 \mathrm{GHz}$. This is due to two factors: the higher emission efficiency of cloud liquid water at $89 \mathrm{GHz}$ than at $37 \mathrm{GHz}$, and the higher sea surface microwave emission at $89 \mathrm{GHz}$ than at $37 \mathrm{GHz}$ (the optical depth of a cumulus or stratocumulus cloud at microwave frequencies is typically less than 0.2 ).

\subsection{ACR data}

The ACR is a scanning Doppler cloud radar capable of providing co- and cross-polarization radar reflectivities at $95 \mathrm{GHz}$ with a $0.56^{\circ}$ beamwidth (Sadowy et al., 1997). It was 


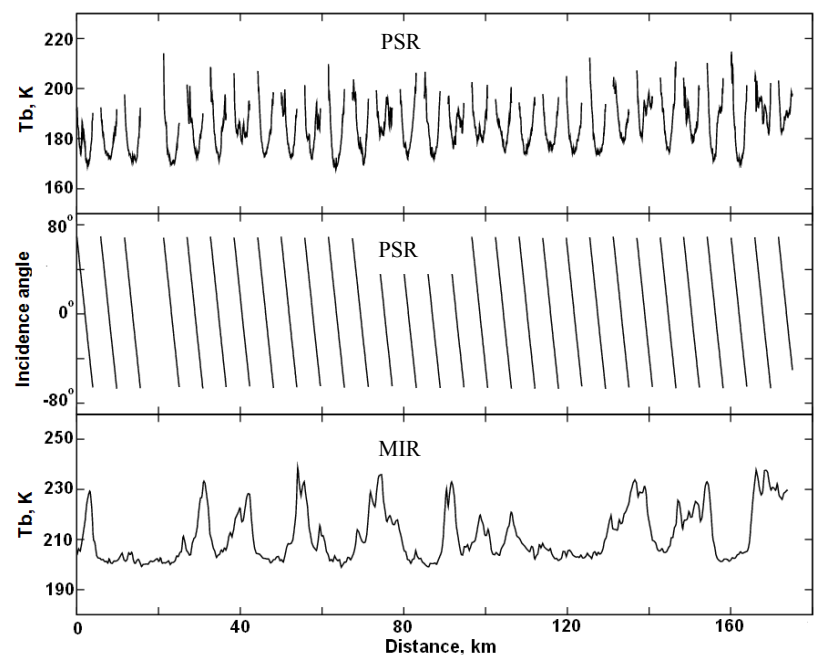

Fig. 4. Examples of radiometric data obtained from the PSR and MIR deployed on the NASA P-3 research aircraft during the Wakasa Bay AMSR-E validation campaign. (a) The PSR brightness temperature as a function of distance; (b) the corresponding viewing angle; (c) the MIR nadir-viewing brightness temperature. The PSR scanned within $\pm 70^{\circ}$ off the nadir along the flight line, providing multi-angular observations of microwave emission in the vertical plane of the flight track. The MIR was programmed to scan within a $106^{\circ}$ swath across the flight track and only the nadir-viewing observations are used in this study.

designed as a prototype airborne facility for the development of the Cloud Profiling Radar System (CPRS), which is the central instrument for NASA CloudSat mission. The ACR can operate in both a fixed (downward and upward looking, nadir parallel) and scanning mode (vertical scanning, various degree intervals off nadir).

During the Wakasa Bay field campaign, the ACR was operated in the fixed downward looking mode. The ACR provides independent observations of two-dimensional cloud structure along the flight track. Thus the ACR data can be used to evaluate the tomographic retrievals from the passive observations of microwave radiometers. The ACR data used in this study were processed by the Department of Atmospheric Sciences of Colorado State University and were made available at NSIDC. Figure 5 shows a 2-D snapshot of radar reflectivity factors obtained within the flight line shown in Figure 2. The radar reflectivity factors range from $-40 \mathrm{dBZ}$ (clear sky return) to $40 \mathrm{dBZ}$ (sea surface return). The maximum reflectivity of the clouds is $15 \mathrm{dBZ}$, suggesting that a significant number of large particles such as rain and ice were present in the clouds. No bright band (melting layer which appears to be brighter to the radar than neighboring layers) is found in the image. This is consistent with the dropsonde measurements that the air temperature at low altitudes is close to the freezing point (more details are provided in Sect. 2.5).

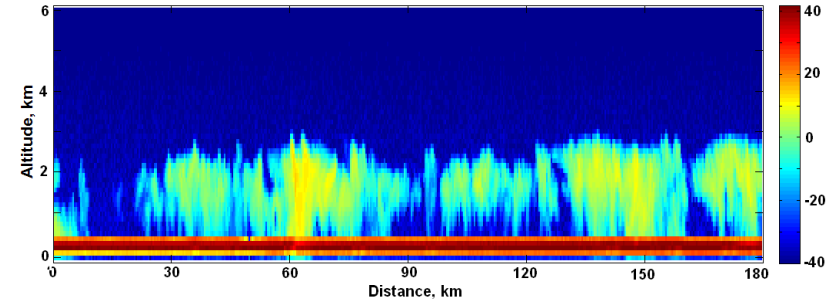

Fig. 5. Image of co-polarization radar reflectivity factor obtained by the ACR. The high values ( $>30 \mathrm{dBZ}$ ) near the surface correspond to the sea surface returns. Below the sea surface, no radar return is allowed. However, multiple scattering of microwave between clouds and the sea surface makes some of the radar returns appear to be from below the sea surface.

\subsection{Dropsonde data}

Various types of radiosondes have been widely used to make direct in-situ measurements of air temperature, humidity and pressure with height, typically to altitudes of approximately $30 \mathrm{~km}$. During the cloud tomography experiment, there was a dropsonde launch every $10-15$ min from the NASA P-3 aircraft. Three of them were within the 176-km long flight line shown in Fig. 2. We average the data from these three dropsondes to obtain the mean profiles of atmospheric temperature and humidity. Figure 6 shows the mean profiles of air temperature and water vapor mixing ratio. The air temperature is close to $0^{\circ} \mathrm{C}$ near the surface and decreases to $-25^{\circ} \mathrm{C}$ at the altitude of $4.1 \mathrm{~km}$ (no dropsonde data available above this level). The relative humidity is $63 \%$ at the surface level, increases steadily to its maximum value of $92 \%$ at the altitude of $1.5 \mathrm{~km}$, and falls gradually to less than $10 \%$ at $4 \mathrm{~km}$. The lifting condensation level and adiabatic cloud water content then can be easily calculated from the mean atmospheric profiles.

\section{Theoretical basis for the inversion problem}

The radiation intensity recorded by microwave radiometers, usually expressed as brightness temperature, is related to the spatial distribution of cloud liquid water and other atmospheric variables through a one-dimensional (1-D) radiative transfer equation. Since cloud droplets appear to be very close to blackbody in the microwave region, the scattering term can be omitted in the radiative transfer equation. Given a total number of $m$ microwave rays, the 1-D radiative transfer equation can be discretized by dividing the retrieval domain into $n=N^{3}$ ( $N^{2}$ for a 2-D slice) equal-sized pixels to yield the following matrix equation:

$\mathbf{A x}=\boldsymbol{b}$,

where $\boldsymbol{x}^{T}=\left(\alpha_{1}, \alpha_{2}, \cdots, \alpha_{n}\right)$ is the vector of atmospheric absorption coefficients (includes contributions from cloud water, water vapor, and oxygen); $\boldsymbol{b}^{T}=\left(b_{1}, b_{2}, \cdots, b_{m}\right)$, is the 


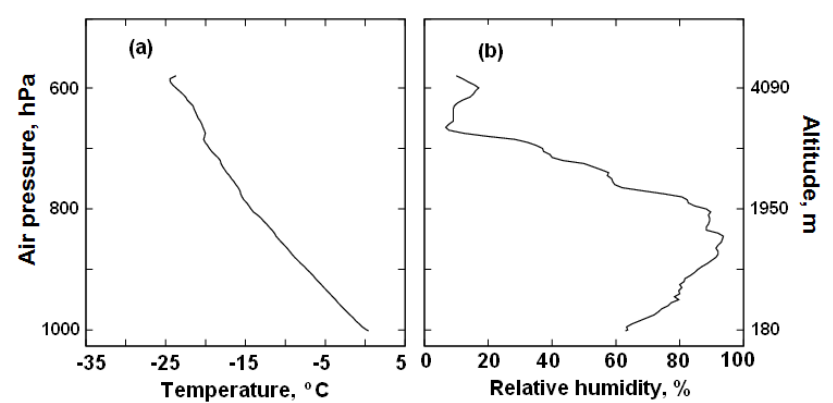

Fig. 6. The mean profiles of air temperature and relative humidity obtained from three dropsondes launched during the flight.

vector of adjusted microwave brightness temperatures; and $\mathbf{A}=\left(a_{i j}\right)$, also called a kernel matrix, is an $m \times n$ matrix that approximates the radiative transfer operator discretely. The kernel matrix A relates the observed brightness temperatures to the atmospheric absorption coefficients.

The tomographic retrieval problem now becomes the inversion of the matrix Eq. (1) to obtain the vector of absorption coefficients and then the absorption coefficients can be easily converted into cloud LWC (Warner et al., 1985; Huang et al., 2008a). While a direct application of the conventional least squares method to an ill-posed problem like cloud tomography would result in large errors in the solution, regularization techniques are usually needed to reduce the sensitivity of the solution to observational noise and numerical errors (Twomey, 1977). In our previous studies (Huang et al., 2008b), we adapted the Tikhonov regularization technique to make use of a variety of constraints. The inclusion of constraints greatly reduces the retrieval sensitivity to noise and numerical errors and thus obtains a better retrieval for the illposed tomographic retrieval problem. The idea is to seek the appropriate vector $\boldsymbol{x}$ for the following constrained minimization problem:

$\min _{x}\left\{\|\mathbf{A} \boldsymbol{x}-\boldsymbol{b}\|_{2}^{2}+\lambda\|\mathbf{L} \boldsymbol{x}\|_{2}^{2}\right\}$,

where the term $\lambda\|\mathbf{L} \boldsymbol{x}\|_{2}^{2}$ is the regularization term, $\|\bullet\|_{2}$ stands for the Hilbert $L_{2}$ norm, $\mathbf{L}$ is the regularization matrix and usually takes the form of a discrete approximation of the spatial derivative operator, and $\lambda$ is the regularization parameter determining the weight of the smoothness to be imposed on the retrieval. It is known that regularization techniques in the form of an $L_{2}$ norm, e.g., the Tikhonov regularization, tend to bias toward a smooth solution (Strong and Chan, 2003). This is confirmed by one of our previous studies (Huang et al., 2008b) that show the Tikhonov method often fails to capture large discontinuities in the cloud LWC field, e.g., the retrieval cannot reproduce the sharp edges at cloud top and instead the retrieved cloud top boundaries are often blurred and extended to higher altitudes.
In order to improve the retrieval of discontinuous structures, non-linear regularization techniques in the form of $L_{1}$ norm such as total variation (TV) regularization have been proposed in image restoration applications (Acar and Vogel, 1994; Chambolle and Lions, 1997):

$\min _{x}\left\{\|\mathbf{A} \boldsymbol{x}-\boldsymbol{b}\|_{2}^{2}+\lambda\|\mathbf{L} \boldsymbol{x}\|_{1}\right\},$.

For the TV regularization, the regularization term can be written as $\|\mathbf{L} \boldsymbol{x}\|_{1}=\mathrm{TV}(\boldsymbol{x}) \equiv \sum_{i}\left|\nabla x_{i}\right| \Delta r \equiv$ $\sum_{i}\left|\left(\frac{\partial}{\partial h}+\frac{\partial}{\partial v}\right) x_{i}\right| \Delta r$, where $r$ denotes the spatial coordinate, $\Delta r$ stands for the area or volume of an individual pixel and can usually be neglected in the practical computation, $h$ and $v$ respectively denote the horizontal and vertical directions. A more detailed discussion on the choice of the regularization term will be presented in the Part 2 of this paper. The main advantage of the $L_{1}$ norm total variation regularization is that it does not penalize more for a larger discontinuity in the solution, while simultaneously it does not bias toward smoothness in the solution; thus under certain conditions it can preserve the exact discontinuous structures in the solution (Acar and Vogel, 1994; Strong and Chan, 2003). However, the implementation of such $L_{1}$ norm regularization techniques is more difficult than the $L_{2}$ norm techniques because of the non-linearity of the $L_{1}$ norm regularization term.

In this research, several other constraints beside the smoothness constraint are also used in the retrieval algorithm. First, to better capture the adiabatic or sub-adiabatic increase of cloud liquid water with height, we make a small modification to the regularization term shown in Eq. (3). The new regularization term is now written as: $\|\mathbf{L} \boldsymbol{x}\|_{1}=$ $\sum_{i}\left|\nabla x_{i}\right| \Delta r \equiv \sum_{i}\left|\left(\frac{\partial}{\partial h}+\frac{\partial}{z \partial v}\right) x_{i}\right| \Delta r$, where $z$ stands for the central height of the volume $\Delta r$. This constraint will usually drive the vertical distribution of retrieved cloud LWC toward an adiabatic shape as long as the solution still satisfies the data constraint. Second, a non-negative constraint is included to meet the apparent physical requirement that all retrievals must be non-negative. The non-negative constraint was also used in our previous cloud tomography retrieval algorithms. Last, a support constraint is imposed on the retrieval; the support constraint forces the retrieval to vanish outside a pre-determined support region S. The use of the support constraint is necessary since some knowledge of cloud boundaries is usually available from a radar image or can be obtained from radiosonde-based atmospheric temperature and humidity profiles. More details on the implementation of these constraints are provided later in this section.

A major difficulty of all the regularization techniques is to determine the weight of the regularization term, in other words, to determine how much information in the retrievals should come from the regularization. Usually the weighting parameter $\lambda$ is determined either in an ad hoc manner 
based on a priori knowledge or in an iterative manner by performing the inversion many times with different regularization parameters and choosing the appropriate regularization parameter based on the L-curve or the cross-validation method. This iterative method for choosing the regularization parameter is computationally expensive and sometimes is difficult to apply in operational algorithms. In practical applications, there is usually an estimate of the instrumentrelated observational error and the forward modeling error, and this information can be used to determine the appropriate regularization parameter. To do this, here we reformulate the regularization problem in a slightly different way with the formulations of Eqs. (2) and (3). For the TV regularization, the new formulation is:

$$
\begin{aligned}
& \min _{x}\left\{\|\mathbf{L} \boldsymbol{x}\|_{1}\right\}, \text { subject to }\|\mathbf{A} \boldsymbol{x}-\boldsymbol{b}\|_{2}^{2} \leq \varepsilon \\
& \quad \text { and other constraints. }
\end{aligned}
$$

Here $\varepsilon$ is the error tolerance of the observations. Hansen (1998) pointed out that for any choice of $\varepsilon$ in Eq. (4) there always exists a regularization parameter $\lambda$ with which the formulation (2) will yield the same solution as (4).

The constrained inversion problem (Eqs. 1-4) can be solved by a direct inversion method, i.e., the method involving the calculation of the inverse of the kernel matrix. The direct method is fast and works well when the dimensionality of the problem is not large. The computational time increases with $O\left(n^{2}\right)$; thus for very high-dimensionality problems the direct method will be very slow and sometimes even impossible because the size of the kernel matrix will become unmanageable. In contrast, for an iterative method like the algebraic reconstruction algorithm (Gordon et al., 1979), a widely used technique in many tomographic applications, the computational cost increases only linearly with the dimensionality of the problem $(O(n))$. Therefore, it has a great advantage over the direct method for large dimensional problems. In this study, we choose the iterative method because of the large number of radiometric observations (about 10000 beams with a 0.15 -s averaging).

Figure 7 shows the pseudo-code that implements the algorithm (4) and it is a modification of the algorithm proposed by Sidky et al. (2008). The aim is to minimize the total variation of the retrieval subject to the data constraint (or the observational constraint), the non-negativity constraint, and the support constraint. The minimization of the total variation term is achieved by a steepest descent method with an adaptive step-size $d_{\mathrm{TV}}$. The other constraints are enforced by the use of projection onto convex sets (POCS) (Youla and Webb, 1982; Sezan and Stark, 1982, 1983). The POCS projection operator for the data constraint, which forces the predicted observations $\mathbf{A} \boldsymbol{x}$ to fall within a specified tolerance $\varepsilon$ of the observed data $\boldsymbol{b}$, is chosen to be the simultaneous algebraic reconstruction technique (SART) with a decreasing step size $d_{\text {data }}$ (Anderson and Kak, 1982). The non-negativity constraint satisfies the apparent physical requirement that cloud

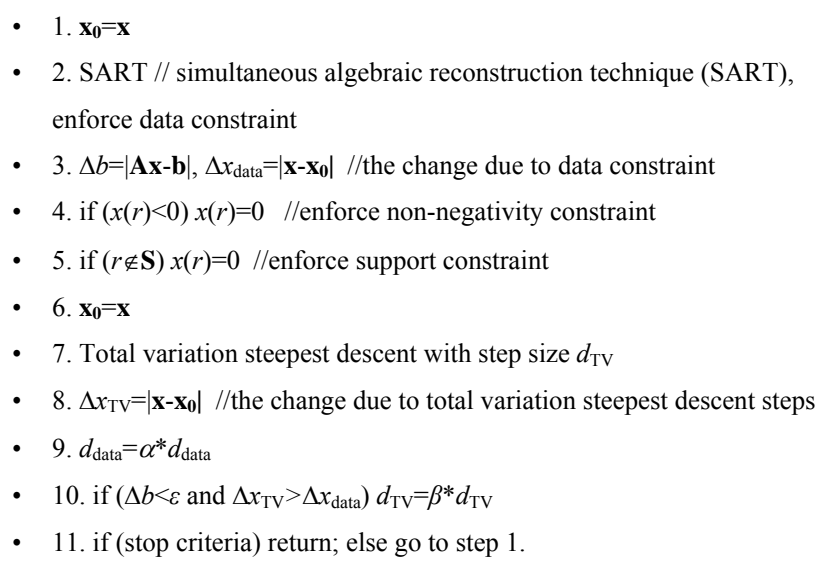

Fig. 7. The pseudo-code of the constrained retrieval algorithm. The constraints implemented in the retrieval algorithm include smoothness, non-negativity, and support constraints. $\boldsymbol{x}$ and $\boldsymbol{b}$ represent the atmospheric absorption coefficients and the observed brightness temperatures. $d_{\mathrm{data}}$ and $d_{\mathrm{TV}}$ are the step sizes for the data constraint and for the total variation steepest descent, respectively. $\alpha$ and $\beta$ are the dampening factors, $0<\beta<\alpha<1$.

water content must be non-negative and the corresponding POCS projection operator takes the simple form shown in line 4 (Fig. 7). The support constraint allows the retrieval to be non-zero only in the support region S and its corresponding POCS projection operator is described in line 5 (Fig. 7).

The step-size adaptation of the TV steepest descent is performed at line 10 as follows: at each iteration the changes of the retrieval due to the data constraint and due to the TV constraint are computed, respectively; the step-size of the TV constraint is adjusted to make sure that the change of the retrieval due to the TV constraint is smaller than that due to the data constraint. This means the retrieval must first satisfy the data constraint (within the observational error tolerance) when moving toward the direction preferred by other types of constraints. At each iteration, the step size for the data constraint $d_{\text {data }}$ is dampened by a factor $\alpha$ that is slightly smaller than unity. When the change of the retrieval due to the TV steepest descent is larger than that due to the data constraint, the gradient-descent step-size $d_{\mathrm{TV}}$ is reduced by a factor $\beta$ that is slightly less than the dampening factor $\alpha$ for the data constraint. Once the retrieval satisfies the datatolerance condition as the iteration proceeds, $d_{\mathrm{TV}}$ will be no longer reduced, allowing it to become larger than $d_{\mathrm{data}}$, because $d_{\text {data }}$ is always decreasing. As a result, the retrieval will drift toward lower-TV directions. When the data constraint is violated again, the steepest-descent step-size reduction will continue. 


\section{Retrieval procedures}

Section 3 not only describes the theoretical formulation of the cloud tomography retrieval problem but also establishes the basis for the constrained retrieval algorithm. In order to use the aforementioned constrained algorithm to solve Eq. (4) to obtain the vector of absorption coefficients, the kernel matrix $\mathbf{A}$ and the observation vector $\boldsymbol{b}$ have to be specified using available observations or model simulations.

First, the microwave emission from the underlying sea surface is needed. The vector $\boldsymbol{b}$ in Eq. (1) represents the "adjusted" microwave observations, that is, the contribution from the background must be substracted from the original microwave observations. The microwave signal received by the airborne radiometers is composed of the thermal emission from the atmosphere along the observation path plus the path-attenuated emission from the underlying sea surface. Therefore, the background emission, i.e., sea surface emission here, has to be specified either through direct measurements (not available in the Wakasa Bay field campaign) or through model simulations. It is well known that sea surface microwave emission depends not only on the thermodynamic temperature of the sea surface but also on the incidence angle. This dependency can be further complicated by waves and foam caused by surface wind. According to the dropsonde record, the wind speed was about $15 \mathrm{~m} / \mathrm{s}$ at the altitude of $175 \mathrm{~m}$ and increased to $25 \mathrm{~m} / \mathrm{s}$ at $2300 \mathrm{~m}$. In this study, a two-scale sea surface thermal emission model (Johnson, 2006) is used to estimate the directional variation of sea surface brightness temperatures at different spectral frequencies.

Second, some slant beams can pass through both the retrieval domain and its two adjacent regions. In order to tomographically retrieve the spatial distribution of cloud LWC in the retrieval domain, the knowledge of cloud LWC distribution in its adjacent regions is needed. The vector $\boldsymbol{b}$ in Eq. (1) should be further adjusted using to a radiative transfer equation so that the contribution from adjacent regions is subtracted. Given the aircraft cruising altitude of $6.7 \mathrm{~km}$ and the maximum off-nadir scanning angle of $70^{\circ}$, a simple geometrical calculation shows that the cloud liquid water distributions in two 15-km long regions, one northeast and the other southwest of the retrieval domain, are needed to calculate the vector $\boldsymbol{b}$. It is clearly unfortunate that there were no other direct measurements of the cloud LWC during the experiment that can be used to specify the cloud fields in the two adjacent regions. So we use the ACR radar reflectivity factors at $95 \mathrm{GHz}$, combined with a simple sub-adiabatic cloud model, to estimate the spatial distribution of cloud LWC in the two adjacent regions. It is known that converting radar reflectivity factors to cloud LWC using empirical Z-LWC relationships (formulas that convert radar reflectivity into cloud LWC) would be unreliable when the radar data are contaminated by large particles like ice and precipitation (Frisch et al., 1995). Also it is difficult to determine the cloud base from radar reflectivity alone if the cloud is drizzling or precipitating. Therefore we use the radar data only to determine the cloud top boundary and set the cloud base to be the lifting condensation level that can be calculated using the atmospheric profiles derived from nearby dropsonde descents. We then use a subadiabatic profile to approximate the vertical distribution of cloud LWC. A subadiabatic profile is characterized by the adiabaticity, defined as the ratio of actual subadiabatic LWP to the adiabatic LWP. As will be discussed later in detail, the adiabaticity is a tunable parameter in the retrieval algorithm and it is determined by minimizing the mean difference between the observed and predicted brightness temperatures.

With the background and side boundary emission specified, the last step is to invert the calibrated radiometric data to obtain the vector $\boldsymbol{x}$ of absorption coefficients (see Eq. 1) using the constrained least squares algorithm described in Sect. 3. The constraint terms in formulation (4) have to be specified. First, we estimate the overall uncertainty in the measured microwave brightness temperature plus the modeled sea-surface brightness temperature is around $2.0 \mathrm{~K}$. The error tolerance $\varepsilon$ is thus set to be $2.0 \mathrm{~m}$, where $m$ is the number of observations. Second, the cloud top height is around $3.0 \mathrm{~km}$ from the radar image (Fig. 5) and this is further confirmed by a temperature inversion observed at $3.1 \mathrm{~km}$ (Fig. 6). Conservatively, we thus set the support constraint to be within $3.5 \mathrm{~km}$, that is, cloud water content will be zero above $3.5 \mathrm{~km}$.

With the constraints being specified, the vector $x$ of absorption coefficient can be obtained by the inversion algorithm described in Fig. 7. The cloud absorption coefficient is proportional to cloud LWC and also depends upon the thermodynamic temperature and the mass of water vapor and oxygen (Warner, et al., 1985); thus the vertical profiles of air temperature, pressure, and water vapor mixing ratio are needed in order to extract the distribution of cloud LWC from the vector $\boldsymbol{x}$ of absorption coefficient. In this study, these necessary profiles are readily obtained from several dropsondes launched during the flight (Fig. 6). The absorption efficiencies of liquid water, water vapor and oxygen are calculated using the absorption model of Rosenkranz (1998).

\section{Results and discussions}

Our previous studies showed that, for a fixed ground-based cloud tomography configuration, the number of radiometers and their physical arrangement determine how well the cloud tomography retrieval can resolve fine spatial structures (Huang et al., 2008a). For the airborne tomography system with a single scanning radiometer, the spacing between successive radiometer scan cycles determines the intersection between microwave beams and thus should have a similar impact on the tomographic retrieval. In order to examine this impact, we perform three retrieval experiments: the first 


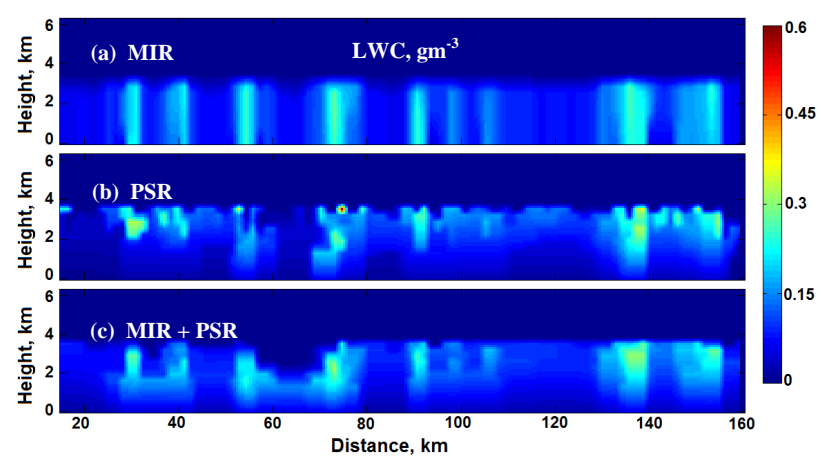

Fig. 8. Retrieved cloud liquid water fields using the MIR data, the PSR data, and the combination of MIR and PSR data.

experiment uses only the nadir-viewing MIR cross-track data (no intersection between beams); the second experiment uses only the PSR along-track data; and the third includes both the MIR nadir data and the PSR data. We then evaluate the tomographic retrievals by examining the consistency between the retrievals and the cloud radar image and by comparing the resultant liquid water path (LWP) from the three retrieval experiments.

\subsection{Retrieval using only the MIR nadir data}

Figure 8a shows the cloud LWC field from the first retrieval experiment (which uses only the MIR nadir data). The mean LWC is $0.069 \mathrm{gm}^{-3}$ and the mean LWP is $462 \mathrm{gm}^{-2}$. The retrieved cloud LWC field appears to be vertically uniform at most locations, revealing almost no information about the vertical structure of clouds. This is not a surprising result because the nadir MIR beams fail to meet the critical requirement of cloud tomography that the beams of different scanning cycles must intersect with each other to some extent. Theoretically, these nadir-viewing observations contain only the path-integrated cloud information, i.e., LWP. The horizontal structure of the MIR LWP is sometimes correlated with vertically integrated radar reflectivity (Fig. 5). The MIR retrieval has little cloud water in the regions $45-55 \mathrm{~km}, 60$ $70 \mathrm{~km}$, and $140-150 \mathrm{~km}$, while the radar shows very strong returns (5-20 dBZ) in these regions which indicates the presence of large particles such as ice. In particular, the scattering of ice in these regions depresses the observed brightness temperatures at $89 \mathrm{GHz}$ and thus depresses the MIR-retrieved LWC (Wang et al., 2005).

The presence of ice in regions of depressed brightness temperature is further confirmed by examining the radar linear depolarization ratio (LDR), defined as the ratio of the power received in the orthogonal, or cross-polarized, channel to that received in the transmitted, or copolarized, channel (Fig. 9). The dominant component of backscattered signal from liquid cloud drops has the same polarization as the transmitted signal, and only large non-spherical parti-

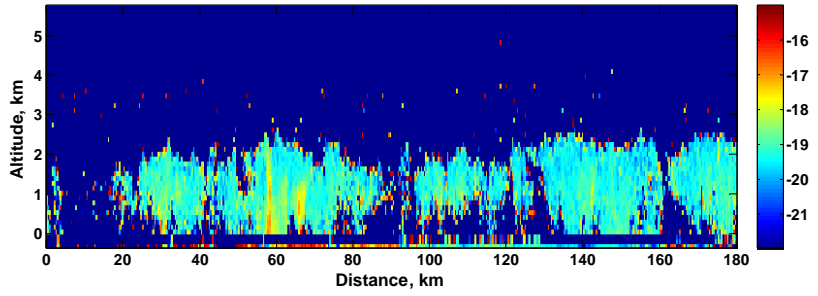

Fig. 9. Image of linear depolarization ratio (LDR) obtained by the ACR. The most probable value of LDR inside the clouds is $-19.2 \mathrm{~dB}$, while bands of higher LDR are also found around locations at $30,58,68$, and $143 \mathrm{~km}$. These high LDR bands are indicative of non-spherical ice particles which can change the polarization of scattered microwave emission. Nevertheless, quantitative retrieval of ice from the LDR data is difficult in this case because the LDR signal is very weak (only $1-1.5 \mathrm{~dB}$ ).

cles, such as ice, can appreciably change the polarization of the transmitted signal. Thus the high LDR bands in Fig. 9 around $30,58,68$, and $143 \mathrm{~km}$ are indicative of ice presence. The locations of these bands are consistent with the bands of brightness temperature depression observed by the MIR. Quantitative retrieval of ice using the depolarization signal is beyond the scope of this paper.

\subsection{Retrieval using only the PSR data}

The retrieval from the second experiment (which uses only the PSR along-track data) shows not only the horizontal variation of clouds but also some vertical structure (Fig. 8b). The maximum LWC in the retrieval is about $0.5 \mathrm{gm}^{-3}$ and is found between 2.0 and $2.8 \mathrm{~km}$ altitude. A fully adiabatic cloud would have a LWC of about $1-1.4 \mathrm{gm}^{-3}$ at these altitudes. Thus the maximum LWC in the retrieved cloud field is $30-40 \%$ of the adiabatic value. This fraction is consistent with many of the in-situ and remote sensing observations of marine low-level clouds (Albrecht et al., 1990; Miller et al., 1998). The spatial characteristics of the retrieved clouds around $30,110,140$ and $160 \mathrm{~km}$ are consistent with those in the radar image (Fig. 5). Some retrieval artifacts are also noticeable. For example, the tomographic retrieval shows large LWC values at $75 \mathrm{~km}$ distance and about $3.5 \mathrm{~km}$ altitude while radar image shows no significant return at this location. We suspect that such artifacts at relatively high altitudes are related to poor beam overlap resulted from the geometric limitation of the airborne tomographic configuration illustrated in Fig. 3.

The PSR retrieval reasonably locates some of the regions with low LWC where the radar reflectivity factors are below $-35 \mathrm{dBZ}$. The cloud top height compares reasonably well with that in the radar image, which seems to be a result of the cloud support constraint described in Sect. 3 that forces the retrieval algorithm to produce little or no clouds at altitudes higher than $3.5 \mathrm{~km}$. The MIR retrieval incorrectly labels the 
regions around 52 and $152 \mathrm{~km}$ as clear sky, while the PSR substantially improves the retrieval in these regions, mainly due to less microwave scattering by ice at $37 \mathrm{GHz}$ than at $89 \mathrm{GHz}$. In the region between $58 \mathrm{~km}$ and $68 \mathrm{~km}$ the radar shows very strong backscattering signal while the PSR retrieval indicates only very thin clouds or clear sky. We suspect, again, that this is due to the strong scattering by ice particles which makes the region appear colder and thus reduces the retrieved cloud LWC.

\subsection{Retrieval using combined MIR and PSR data}

For the third retrieval experiment with both the MIR and PSR data (Figure 8c), several improvements are noticeable, although, at first glance, the retrieved spatial distribution of cloud LWC looks similar to that from the second experiment (which uses only the PSR). Around 35 and $105 \mathrm{~km}$, the retrieval from the third experiment shows more consistent cloud structures compared to the radar image than the retrieval from the second experiment. The regions with low cloud LWC are better resolved. The reason for these improvements is as follows: the MIR scans through the nadir every three seconds, while the PSR provides a nadir view every $44 \mathrm{~s}$ when it is operated in along-track scan mode. Thus the addition of the MIR data improves the horizontal cloud structure in the retrieved cloud field.

It should be pointed out that the tomographic data obtained during the Wakasa Bay experiment do not contain enough information to determine cloud structure at high altitudes. We find that, when no cloud support constraint is used, some vertically-uniform puffy clouds appear at high altitudes in the retrieved cloud LWC field, while the radar image shows only clear sky at the same altitudes. These high-altitude clouds are physically implausible because, if present at such high altitudes, they would be dominated by ice particles that are almost invisible to centimeter wavelength radiometers. The vertically-uniform clouds appearing at high altitudes in the retrieval are likely to be caused by the insufficient overlap between the swaths of successive PSR scan cycles. As shown in Fig. 3, due to the high speed of the NASA P-3 aircraft there is almost no overlap between the swaths of two successive scans at altitudes higher than $4 \mathrm{~km}$; thus the resultant radiometric data contain almost no information on the spatial distribution of cloud water there.

\subsection{Liquid water paths calculated from the retrievals}

The retrieved LWC fields in the three experiments are integrated vertically to obtain the LWP at each location along the flight track. Figure 10a shows the point-by-point comparison between the MIR LWP and the PSR LWP. The correlation coefficient is 0.96 , suggesting consistency between the MIR and the PSR retrieved horizontal structures. The mean MIR LWP averaged along the flight track also agrees well with that of the PSR. The slope of the regression line is signifi-
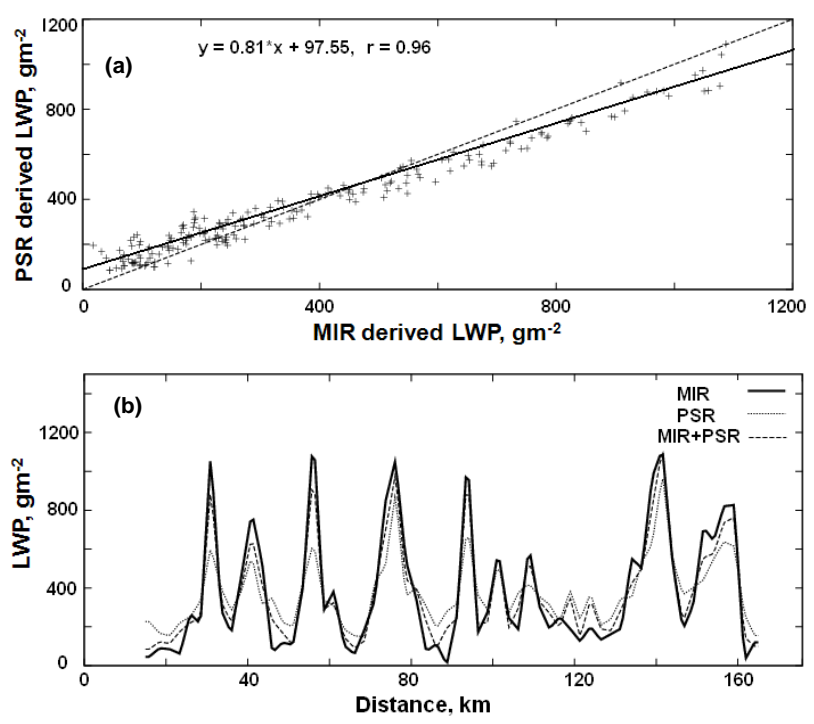

Fig. 10. Comparison of the liquid water paths based on the MIR and PSR data: (a) scatter plot with the robust regression line (solid) and the line of equal LWPs (dash); (b) LWPs as a function of distance.

cantly smaller than 1.0 and the intercept is about $100 \mathrm{gm}^{-2}$. According to Fig. 10a and b, the MIR LWP values are significantly higher than those of the PSR for regions that have thick clouds $\left(\mathrm{LWP}>500 \mathrm{gm}^{-2}\right)$, while, for regions with relatively thin clouds $\left(\mathrm{LWP}<150 \mathrm{gm}^{-2}\right.$ ), the MIR LWP values are consistently lower than those of the PSR. Thus, the distribution of PSR LWP appears to be much narrower than that of the MIR LWP. There may be several reasons for this.

First, the finest scale that the cloud tomography method can resolve depends on the total number of beams and on the extent to which these beams intersect (Huang et al., 2008a). Beam intersection is determined both by the radiometer scanning speed and the aircraft speed. Each scan cycle of PSR takes $44 \mathrm{~s}$ and during this period the aircraft moves about $6 \mathrm{~km}$, resulting in moderate overlap between two successive scans but very little overlap between a scan and its second previous scan (Fig. 3). So we expect relatively poor horizontal and vertical resolution for the PSR retrieval. On the other hand, the MIR scanned a $106^{\circ}$ swath across the flight track every three seconds, producing a nadir observation every 400 $\mathrm{m}$ along the track. Thus, the MIR retrieval has an along-track (horizontal) resolution of about $400 \mathrm{~m}$.

The second reason for the narrower distribution of the PSR LWP is that the regularization techniques in the retrieval algorithm, especially the use of a smoothness constraint to reduce the high retrieval sensitivity to noise, may artificially smooth the horizontal structure in the PSR retrieval. This smoothness constraint eliminates extreme values. 

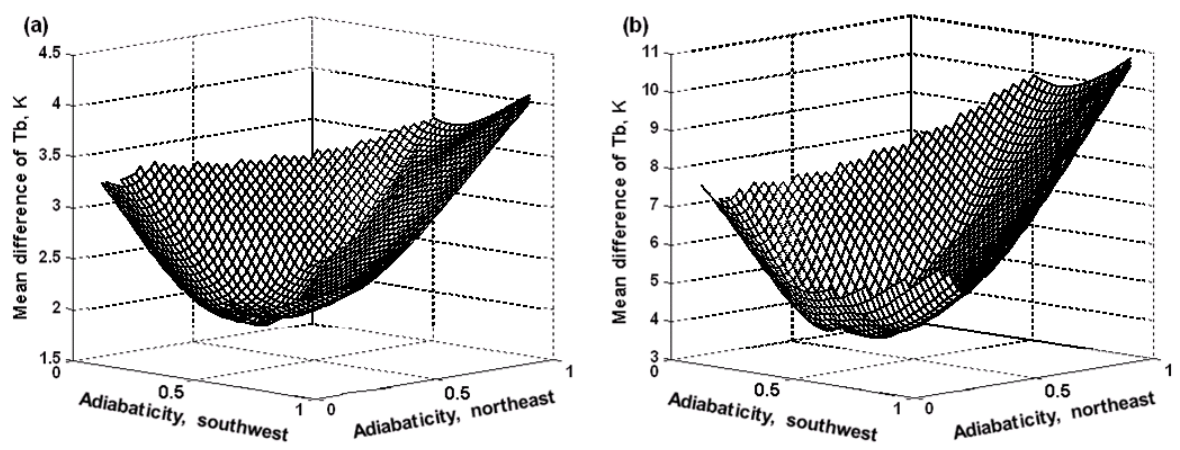

Fig. 11. The mean differences between the calculated and observed brightness temperatures as a function of the adiabaticity of the two regions adjacent to the retrieval domain: (a) for all beams; (b) for beams that pass through either of the two adjacent regions.

\subsection{Mean difference between measured and calculated brightness temperatures}

Now, in a kind of closure experiment, let us compare the measured brightness temperatures with those calculated from the retrieved cloud LWC fields using a radiative transfer model (see Sect. 2 in Part 2 of this paper); this gives a measure not only of how well the retrieval reproduces the given microwave brightness temperature data but also of how accurate the guess of liquid water distribution in the two adjacent regions is. As shown in Sect. 4, these adjacent regions are north-east and south-west of the retrieval domain and are $15 \mathrm{~km}$ wide and $6.7 \mathrm{~km}$ high. Here, a subadiabatic profile is used to approximate the vertical distribution of cloud LWC in these two regions. We then vary the adiabaticity from 0 to 1 and select, as the best guess, the value that gives minimum difference between the measured and calculated brightness temperatures.

Figure 11a shows the 3-D plot of the mean difference between the calculated and PSR-observed brightness temperatures as a function of the adiabaticity of the aforementioned two adjacent regions. The minimum difference between the two sets of brightness temperatures is $1.9 \mathrm{~K}$; this is comparable with the sum of instrumental noise and uncertainty in the forward modeling of the sea surface brightness temperatures. This minimum is found at an adiabaticity of 0.5 in the northeastern region and 0.25 in the southwestern region. When moving away from the minimum point, the mean difference of brightness temperatures increases smoothly from $1.9 \mathrm{~K}$ to $4.1 \mathrm{~K}$. Figure $11 \mathrm{~b}$ is similar to Fig. 11a but calculated using only the microwave beams that pass through either of the two adjacent regions. The minimum difference between the calculated brightness temperatures (for the beams that pass through either of the two regions) and the observed values is $3.3 \mathrm{~K}$ and is found at almost the same adiabaticity values as Fig. 11a, i.e., an adiabaticity of 0.5 in the northeastern region and 0.25 in the southwestern region. This agreement suggests that the guess of cloud LWC fields for the two adjacent regions are consistent with the observed brightness temperatures.

\section{Conclusions}

This paper examined the results from a limited cloud tomography experiment conducted over Wakasa Bay, Sea of Japan, as part of the 2003 AMSR-E validation campaign. During the tomographic experiment, several multi-wavelength microwave radiometers were deployed on the NASA P-3 aircraft and scanned through a system of winter clouds. The aircraft also carried a nadir-pointing W-band cloud radar. The radiometer scanning strategy provides suitable geometry for tomographic retrieval of 2-D cross sections of cloud LWC. To handle the highly ill-posed tomographic retrieval problem, we adapted a constrained inversion algorithm that uses several different types of constraints to obtain more physically plausible retrievals. The constraints used in this research include smoothness, non-negativity, and support (the support constraint forces the retrieval to vanish outside a certain domain).

We performed three retrieval experiments using the constrained inversion algorithm: one with only the MIR nadir data, one with only the PSR along-track scanning data, and one with the combined PSR and MIR data. The retrieval based on the MIR nadir data, as one would expect, provides only vertically-integrated cloud liquid water and thus no information on the vertical structures. The PSR retrieval shows physically plausible cloud structures; it not only reasonably captures the locations of clouds but also reasonably reproduces some features of the cloud vertical profiles. Combining the PSR and MIR data further improves the retrieval at regions with low cloud liquid water. When the support constraint is not included, some implausible results are identified in the retrieval, e.g., vertically-uniform puffy clouds appear at high altitudes where the radar shows no significant return (clear sky), indicating insufficient information in the radiometric data to retrieve cloud structure at such high altitudes.

There were no in-situ measurements of cloud LWC during the field campaign to validate the tomographic retrievals. Therefore, we fell back on three less satisfactory ways of testing our retrievals. First, we compared the retrievals with the radar reflectivity image to qualitatively evaluate how 
well the tomographic method captures the spatial patterns of clouds. The spatial distribution patterns of cloud LWC appear to be consistent with the patterns of radar reflectivity except for regions where we strongly suspect the presence of ice. Second, we calculated the LWP from the retrievals of each experiment and compared the resultant LWPs at each location. The correlation between the PSR LWP and the MIR LWP is 0.96 for the flight leg. The mean PSR LWP along the flight track agrees closely with the MIR LWP, while the histogram of the PSR LWP is much narrower than that of the MIR LWP. Third, we calculated the microwave brightness temperatures corresponding to the retrieved cloud LWC values, consistent with the way the radiometers observe. The mean difference between the calculated brightness temperatures and the observed values is $1.9 \mathrm{~K}$, which is close to the uncertainty associated with the instrumental noise and the modeling of the background sea surface emission. Overall, the consistency between the different types of observations shows the self-consistency and potential of the cloud tomography technique.

The Wakasa Bay field campaign was designed to provide data for validating the precipitation retrieval algorithms developed for the AMSR-E sensor. As a result, many conditions were not ideal for the cloud tomography technique. The NASA P-3 aircraft flew at $144 \mathrm{~m} / \mathrm{s}$, resulting in insufficient overlap between successive scan cycles at high altitudes, which leads to ambiguity in the retrievals at these high altitudes. The surface wind was strong during the cloud tomography test, causing at least a $2-3 \mathrm{~K}$ modeling error of background (sea surface) brightness temperatures. This modeling error will inevitably propagate into the tomographic retrievals. Nevertheless, this research has demonstrated the potential of tomographically retrieving cloud structure using current scanning microwave radiometer technology and has identified several limitations of the airborne cloud tomography experiment during the Wakasa Bay field campaign. More rigorous sensitivity studies are required to provide guidelines to improve future field-based studies of cloud tomography. This can be achieved by using a suite of complicated field tests that are expensive to implement, or by using observation system simulation experiments that are much easier and cheaper to implement. The latter is the focus of Part 2 of this paper.

Acknowledgements. This work is supported by the DOE Atmosphere Radiation Measurement program under Contract DE-AC02-98CH10886. The authors thank Bob Webber of the University of Colorado at Boulder for providing the two-scale sea surface thermal emission model and helping in setting up the model. We are grateful to Ed Westwater of the University of Colorado at Boulder for many insightful discussions that helped to improve this study. We are grateful to James Wang of the NASA Goddard Fight Center for providing the MIR data and to Richard Austin of Colorado State University for providing the ACR images. We thank Elena Lobl University of Alabama in Huntsville for her help in accessing the dropsonde data. It is a pleasure to acknowledge insightful discussions with Yangang Liu and Michael Jensen of Brookhaven National Laboratory and Guosheng Liu of Florida State University. We thank two reviewers for constructive comments that improved the manuscript.

Edited by: W. E. Asher

\section{References}

Acar, R. and Vogel, C. R.: Analysis of total variation penalty methods, Inv. Prob., 10, 1217-1229, 1994.

Albrecht, B. A., Fairall, C. W., Thomson, D. W., White, A. B., Snider, J. B., and Schubert, W. H.: Surface-based remote sensing of the observed and the adiabatic liquid water content of stratocumulus clouds, Geophys. Res. Lett., 17, 89-92, 1990.

Andersen, A. H. and Kak, A. C.: Simultaneous algebraic reconstruction technique (SART): A superior implementation of the ART algorithm, Ultrason. Imag., 6, 81-94, 1984.

Chambolle, A. and Lions, P. L.: Image recovery via total variation minimization and related problems, Numer. Math., 72, 167-188, 1997.

Corbella, I., Gasiewski, A. J., Klein, M., Leuski, V., Francavilla, A. J., and Piepmeier, J. R.: On-board Accurate Calibration of DualChannel Radiometers Using Internal and External References, IEEE Trans. Microwave Theory Tech., 50, 1816-1820, 2002.

Frisch, A. S., Fairall, C. W., and Snyder, J. B.: Measurement of stratus cloud and drizzle parameters in ASTEX with a Ka-band Doppler radar and a microwave radiometer, J. Atmos. Sci., 52, 2788-2799, 1995.

Gordon, R., Bender, R., and Herman, G. T.: Algebraic Reconstruction Techniques (ART) for Three-dimensional Electron Microscopy and X-ray Photography, J. Theor. Biol., 29, 471-481, 1970.

Hansen, P. C.: Rank deficient and ill-posed problems: Numerical aspects of linear inversion, SIAM, Philadelphia, 247 pp., 1998.

Huang, D., Liu, Y., and Wiscombe, W.: Determination of cloud liquid water distribution using 3D cloud tomography, J. Geophys. Res., 113, D13201, doi:10.1029/2007JD009133, 2008a.

Huang, D., Liu, Y., and Wiscombe, W.: Cloud tomography: Role of constraints and a new algorithm, J. Geophys. Res.-Atmos., 113, D23203, doi:10.1029/2008JD009952, 2008.

Huang, D., Gasiewski, A., and Wiscombe, W.: Tomographic retrieval of cloud liquid water fields from a single scanning microwave radiometer aboard a moving platform - Part 2: Observation system simulation experiments, Atmos. Chem. Phys., 10, 6699-6709, doi:10.5194/acp-10-6699-2010, 2010.

Johnson, J. T.: An efficient two-scale model for the computation of thermal emission and atmospheric reflection from the sea surface, IEEE T. Geosci. Remote., 44, 560-568, 2006.

Lobl, S. E., Aonashi, K., Griffith, B., Kummerow, C., Liu, G., Murakami, M., and Wilheit, T.: Wakasa Bay - An AMSR Precipitation Validation Campaign, B. Am. Meteorol. Soc., 88, 551-558, 2007.

Miller, M. A., Jensen, M. P., and Clothiaux, E. E.: Diurnal cloud and thermodynamic variations in the stratocumulus transition regime: A case study using in situ and remote sensors, J. Atmos. Sci., 55, 2294-2310, 1998.

Padmanabhan, S., Reising, S. C., Vivekanandan, J., and IturbideSanchez, F.: Retrieval of atmospheric water vapor density with 
fine spatial resolution using 3-D tomographic inversion of microwave brightness temperatures measured by a network of scanning compact radiometers, IEEE T. Geosci. Remote, 47, 37083721, 2009.

Piepmeier, J. R. and Gasiewski, A. J.: Polarimetric scanning radiometer for airborne microwave imaging studies, Proceedings of International Geoscience and Remote Sensing Symposium, Lincoln, 1688-1691, 1961.

Racette, P. E., Adler, R. F., Wang, J. R., Gasiewski, A., Jackson, D. M., and Zacharias, D. S.: An Airborne Millimeter Wave Imaging Radiometer for Cloud, Precipitation, and Atmospheric Water Vapor Studies, J. Atmos. Ocean. Tech., 13, 610-619, 1996.

Ramanathan, V., Cess, R., Harrison, E., Minnis, P., Barkstrom, B., Ahmad, A., and Hartmann, D.: Cloud-Radiative Forcing and Climate: Results from the Earth Radiation Budget Experiment, Science, 243, 57-63, 1989.

Rosenkranz, P. W.: Water vapor microwave continuum absorption: A comparison of measurements and models, Radio Sci., 33(4), 919-928, doi:10.1029/98RS01182, 1998.

Sadowy, G., McIntosh, R., Dinardo, S., Durden, S., Edelstein, W., Li, F., Tanner, A., Wilson, W., Schneider, T., and Stephens, G.: The NASA DC-8 airborne cloud radar: Design and preliminary results, Proceedings of International Geoscience and Remote Sensing Symposium, Singapore, 1997.

Sezan, M. I. and Stark, H.: Image Restoration by the Method of Convex Projections: Part 2 - Applications and Numerical Results, IEEE Transaction on Medical Imaging, MI-1, 95-101, 1982.
Sezan, M. I. and Stark, H.: Image restoration by convex projections in the presence of noise, Appl. Optics, 22, 2781-2789, 1983.

Sidky, E. Y. and Pan, X.: Image reconstruction in circular conebeam computed tomography by constrained total-variation minimization, Phys. Med. Biol., 53, 4777-4807, 2008.

Strong, D. and Chan, T. F.: Edge-preserving and scale-dependent properties of the total variation regularization, Inverse Problems, 19, 165-187, 2003.

Twomey, S.: Introduction to the mathematics of inversion in remote sensing inversion and indirect measurements, Elsevier, Amsterdam, 243 pp., 1997.

Wang, J., Racette, P., Austin, R. T., Liu, G. S., and Sekelsky, S. M.: Remote Measurements of Snowfalls in Wakasa Bay, Japan with Airborne Millimeter-wave Imaging Radiometer and Cloud Radar, Proceedings of IEEE International Geoscience and Remote Sensing Symposium, Anchorage, Alaska, 2004.

Warner, J., Drake, J. F., and Krehbiel, P. R.: Determination of cloud liquid water distribution by inversion of radiometric data, J. Atmos. Ocean. Tech., 2, 293-303, 1985.

Warner, J., Drake, J. F., and Snider, J. B.:: Liquid water distribution obtained from coplanar scanning radiometers, J. Atmos. Ocean. Tech., 3, 542-546, 1986.

Youla, D. C. and Webb, H.: Image restoration by the method of convex projections: Part 1 - Theory, IEEE Transaction on Medical Imaging, MI-1, 81-94, 1982. 